\title{
Novel Technique of Radar Interferometry in Dynamic Control of Tall Slender Structures
}

\author{
Tomasz Owerko, Łukasz Ortyl, Rafał Kocierz and Przemysław Kuras \\ Department of Engineering Surveying and Civil Engineering, Faculty of Engineering Surveying and Civil Engineering, AGH \\ University of Science and Technology, Kraków 30-059, Poland
}

\begin{abstract}
Structures of slender shape, like guyed masts, chimneys or TV towers, are exposed to the excitation of dynamic movements. The most common cause of vibration is the wind that induces generation of vortices. Another important reason is a sudden strike from seismic or para-seismic forces. Structure exposed to wind impact is subjected to harmonically various force, perpendicular to the air stream, which is an effect of vortices, shedding on alternate sides of structure. Because of the possibility of vibration the damping of a structure has to be designed. Measuring of decay in the oscillation allows to find the logarithmic decrement of the real structure and compare it with the designed value. Apart from damping, the proper designing of tall slender structures has to preserve the significant difference between vortex shedding frequencies and the natural frequencies of the structure shape modes. In the case of guyed masts it is important to analyse the response of a real structure to the exceptional dynamic load, such as a sudden break of guy or fall off a load from a structure, e.g., icing or antennas. The dynamic analysis of a real structure requires the accurate values describing the dynamic behavior of tall structures (e.g., amplitudes). The accuracy of $0.1 \mathrm{~mm}$ is provided by ground-based interferometric radar. This device is a part of the IBIS-S (image by interferometric survey) system, which calculates the displacement values on the basis of the difference between phases of waves received in consecutive samples. Typically, the measurements of tall structures are performed with several sensors (strain gauges, accelerometers). Instead, the IBIS-S system allows the quasi-continuous (not limited to points) observation of the entire structure without installation of any sensors or reflectors. The real resolution (along the structure), which means the minimum distance between two observed points, amounts up to about $0.7 \mathrm{~m}$.
\end{abstract}

Key words: Vibration, radar interferometry, frequency of vibration, chimneys, masts.

\section{Introduction}

Description of high-dynamic behaviour of engineering structures is an important issue from a security point of view for this type of construction. In the case of high telecommunication mast, structure is subjected to atmospheric influences (wind, icing and rain). In addition, a high degree of the surface development by additional antennas (wireless IP networks, digital radio/TV emission) causes a significant change in its dynamic characteristics. In the case of chimneys, the factors listed above must also be taken into consideration. Moreover, intense impact of gases on the structure's shell is an important factor.

Corresponding author: Tomasz Owerko, assistant professor, research fields: surveying engineering and civil engineering. E-mail: owerko@agh.edu.pl.
Measuring of high structures by classical indirect methods is associated with an acceptance of restrictions imposed by their industrial location. Selection of the measuring stations at industrial plants (steelworks, power plant) is significantly limited. In the case of telecommunication masts it is also not easy to choose such a measuring position to be able to carry out monitoring of the entire structure at once. During extremely adverse conditions (icing, strong wind and low temperature) direct access to this kind of structures may be impossible. In the case of direct techniques safety zones associated with strong electromagnetic fields become a serious restriction of access to mast's facilities.

The issue of determining the dynamic response of tall engineering construction is the subject of many articles and scientific studies. The subjects of analysis 
are frequencies of vibration of slender structures supported by guy ropes and cables [1-3]. A very large number of publications present the theoretical analysis verified on laboratory models of such objects [4]. In some works, based on measurements of real objects, many different sensors were installed. Among them accelerometers are the fundamental measurement devices [5]. The intention of the authors of this paper is to review and present capabilities of the IBIS-S ground-based radar interferometer, which is a relatively new tool for this kind of measurement. The main advantage of radar over other sensors is obviously the lack of necessity of entry into the object (mast or chimney). The result is full information about the object behaviour. Many types of such structures give the opportunity to observe several points along its entire length (height). The paper presents results from IBIS-S in confrontation with accelerometers during tests of the telecommunication mast. In addition, paper presents the results obtained for a 300-metres high industrial chimney.

\section{Subject of Measurement}

The Malczewski Radio Transmit Centre, which operates using the examinated mast, is located in Kraków, Poland $\left(50^{\circ} 03^{\prime} 01\right.$ 'N, 1954'06”E). The structure is 123 metres high (Fig. 1).

The object of measurement was a structure consisted of a truss of a triangular cross-section and guy ropes. It is a three module mast with independent anchoring of each guy rope. The mast consists of three spans and a single segment. Assuming that in this part of Poland the wind of western direction is dominant, measurement tests were designed to capture the maximum amplitude of vibration. Thus the measurement stations were located on the north, south and east from the mast. Bearings of measuring lines were mutually perpendicular.

Another measured object was the industrial chimney located in the power plant Jaworzno III (Fig. 2). The area surrounding the chimney is built with industrial

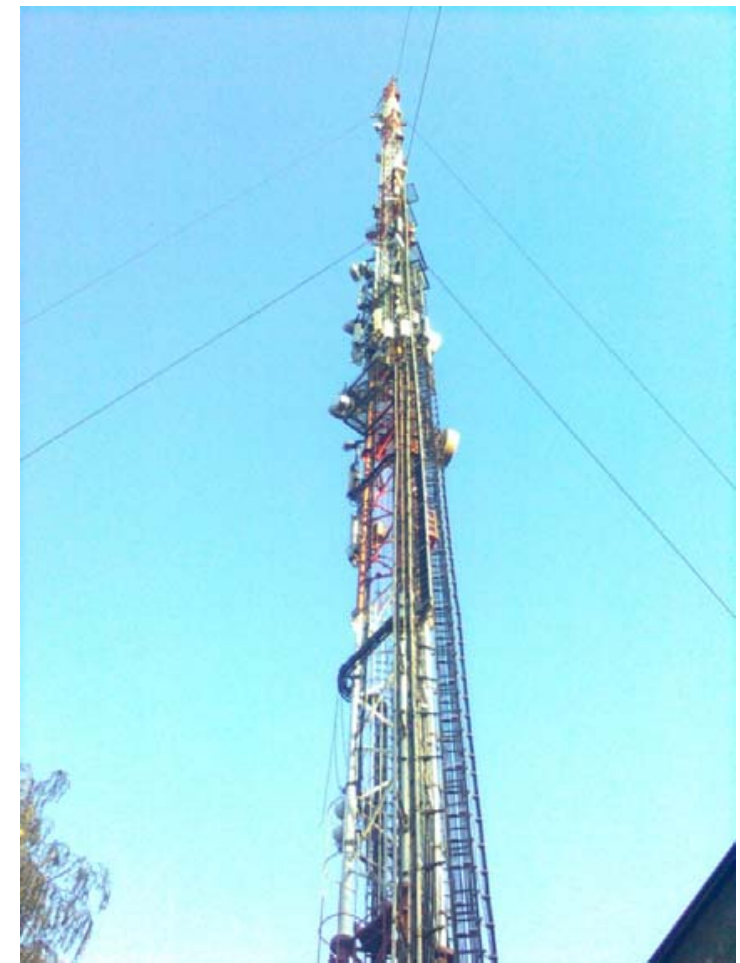

Fig. 1 The Malczewski radio mast.

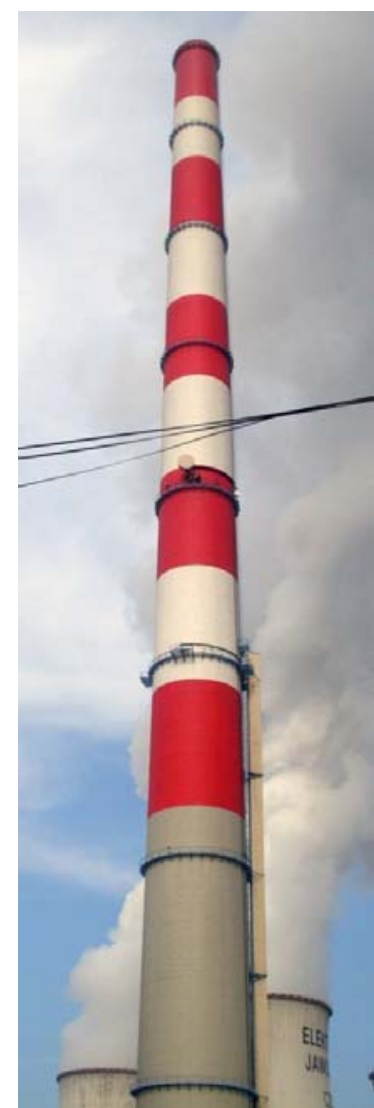

Fig. 2 The industrial chimney located at the power plant Jaworzno III. 
facilities, therefore, the position of instrument was determined by availability of free space needed to carry out the measurement. The observation was performed with two mutually perpendicular directions.

The main reason which causes vibration of chimneys is Karman vortices, especially when the frequency of their shedding on the leeward side equals or approaches one of the natural frequencies of the structure. In the case of circular structures this phenomenon gains strongly in contrast to the truss masts on which multiple antennas are mounted.

\section{Measurement Method}

IBIS system (image by interferometric survey) was developed by the Italian company IDS to monitor the movements of masses of land and/or engineering structures. The IBIS-S version is used to measure displacements of buildings, of which one dimension is significantly larger than the others, such as bridges or towers.

The IBIS-S system includes:

- radar unit-active radar that generates, transmits and receives electromagnetic waves from the scope of the $K_{u}$ band (the wave frequency is around $17 \mathrm{GHz}$ );

- notebook with the software controlling operation of the radar, communicating with radar via USB interface;

- transmitting and receiving antennas ("horns") of defined characteristics of radiation (Fig. 3);

- $12 \mathrm{~V}$ battery pack, enabling work in field.

The bandwidth range used by the radar is $B=300$ $\mathrm{MHz}$, which allows the maximum resolution of $R=0.5$ $\mathrm{m}$. The concept of resolution should be understood as the minimum distance between two points on the object at which they may be considered as different points. This means the opportunity to observe each object point separated by not less than $50 \mathrm{~cm}$ from another point along the radial direction, i.e. the direction of wave propagation (Fig. 4). If the distance is less than $R$, the points will be treated as one. The distance is taken along the direction of wave propagation.
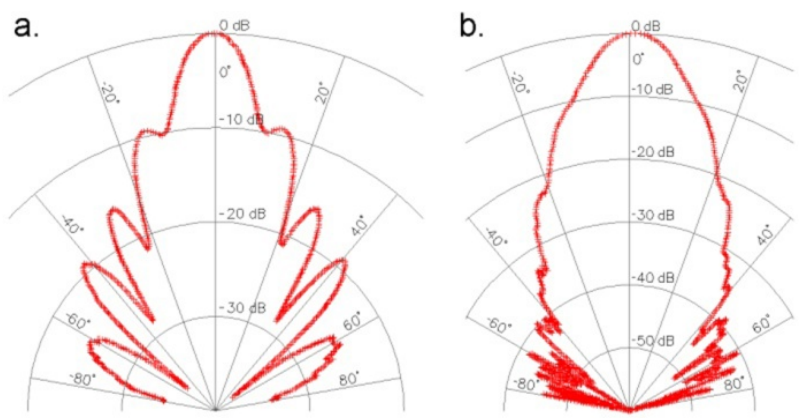

Fig. 3 Plane patterns of antenna of 23.5 DBI maximum gain: a. horizontal; b. vertical.

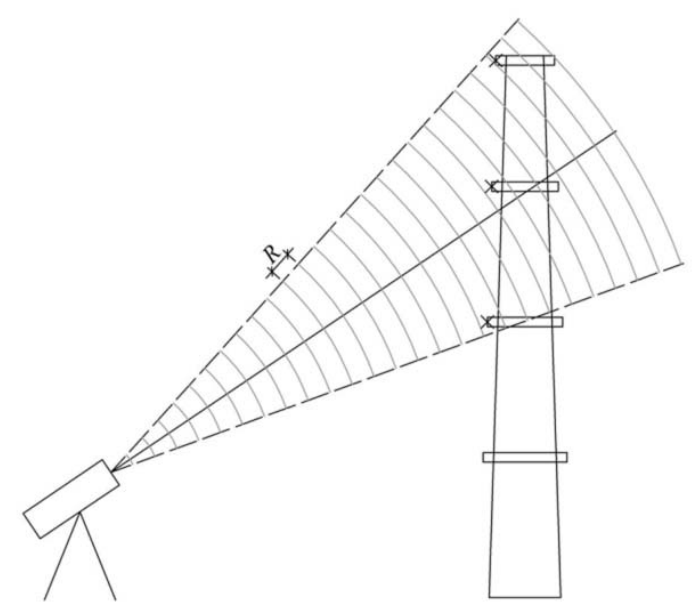

Fig. 4 Diagram of radial resolution.

Parameters that allow to use the IBIS-S radar in displacements measurements are the following:

- recording frequency of all observed points position: up to $200 \mathrm{~Hz}$ [6];

- measurement range: up to $1,000 \mathrm{~m}$ [7];

- accuracy of the radial component of displacement: $0.1 \mathrm{~mm}[8]$.

Resolution, recording frequency and measurement accuracy are mutually dependent and it is not possible to work with maximum values of these parameters simultaneously.

The IBIS-S system is based on two radar techniques:

- microwave interferometry;

- stepped-frequency continuous wave modulation.

Microwave interferometry technique allows to achieve high accuracy measurement of displacement. Point displacement is calculated based on the phase difference of waves received by the receiver at different times. Movement of point in the direction of 
electromagnetic wave propagation induces a phase shift between the signals reflected from the surface of the object. The value of displacement $d$ along the direction of wave propagation can be written as: $d=$ $(\lambda \cdot \Delta \varphi) /(4 \pi)$, where $\lambda$ is the length of electromagnetic wave, and $\Delta \varphi$ is the phase shift.

Stepped frequency continuous wave technique is used to avoid installation of multiple units of measurement on the structure. The corresponding output signal allows to obtain an image displacement of many points (virtual sensors) on the object. In fact, small "inhomogeneities" of the object, on which the wave is scattered, are measured.

Signal used in radar has the form a short pulse. The shorter the pulse duration $\tau$, the higher measurement resolution $R$ can be obtained. This follows from the relation $R=c \cdot \tau / 2$, where $c$ is the speed of light in vacuum. The relationship between pulse duration $\tau$ and bandwidth $B$ used of the microwave range can be written as $\tau \cdot B=1$, and therefore the resolution of the radar is $R=c /(2 B)$.

Increasing the resolution of measurement is achieved by reducing the value of $\tau$ or increasing the value of $B$. Radars SFCW, instead of using short pulses, receive bandwidth through a wide range of discrete, linear increase in the frequency of discrete values of $\Delta f$. Bandwidth can be expressed as: $B=(N-1) \cdot \Delta f$, where $N$ is the number of different frequencies which are within the bandwidth $B$.

In the test two accelerometers based on MEMS (Micro Electro-mechanical Systems) technology, manufactured by the SEQUOIA company, were used. Generally, MEMS combines electrical and mechanical components made in a very small scale to minimize the size and energy requirements of measuring devices. In the case of accelerometers micro-capacitors are used. Miniaturized free mass in the form of a flat plate is one of the conductive plates. Displacement of the movable plate that represents the free mass gives change of the voltage applied to the conductive plate. Spaces between plates as well as their size are about single micrometer. The measurement is carried out based on several similar capacitors which allow averaging the results of observation. Such free mass arranged in perpendicular directions make it possible to determine the accelerations in 3D space. This kind of accelerometer was used in this measurement test.

External dimensions of the Fast Tracer device are $30 \times 55 \times 15 \mathrm{~mm}$ and its mass is 55 grams. The maximum value of determined acceleration is $\pm 5 \mathrm{~g}$. Accelerometer performs 8,192 samples per second. Measured resolution of acceleration is $0.0025 \mathrm{~m} / \mathrm{s}^{2}$ and noise ranges within $0.075 \mathrm{~m} / \mathrm{s}^{2}$. The determined values cover the range of vibration frequencies from 0 to 2,500 MHz. Recording is implemented on a PC via USB or with wireless connection.

Measuring methods which include contactless radar interferometry does not pose problems if the radar profile for the desired points gives a good signal to noise ratio. Basically in the case of truss structures, to which the Malczewski mast belongs, such problems do not occur. Selection of observed points is limited only by comfortable site. On the other hand, direct methods require physical access to the structure and allow for precise data acquisition. The only condition is the ability to mount sensors in the selected location. Fig. 5 presents a place of accelerometers which were mounted in the middle of the central span and on top of the mast. Red color indicates dangerous areas due to the high intensity of electromagnetic field. For installation of accelerometers powerful neodymium magnets were used. Data were obtained from the local wireless network (each accelerometer was equipped with a WiFi gateway).

In the same way, but without the use of accelerometers, measurements were made on an industrial chimney in Jaworzno. As in the case of mast there was no need for additional radar signal intensification. Comparison of the radar profile with a total station measurement allowed for easy identification of measurement points on the chimney. 


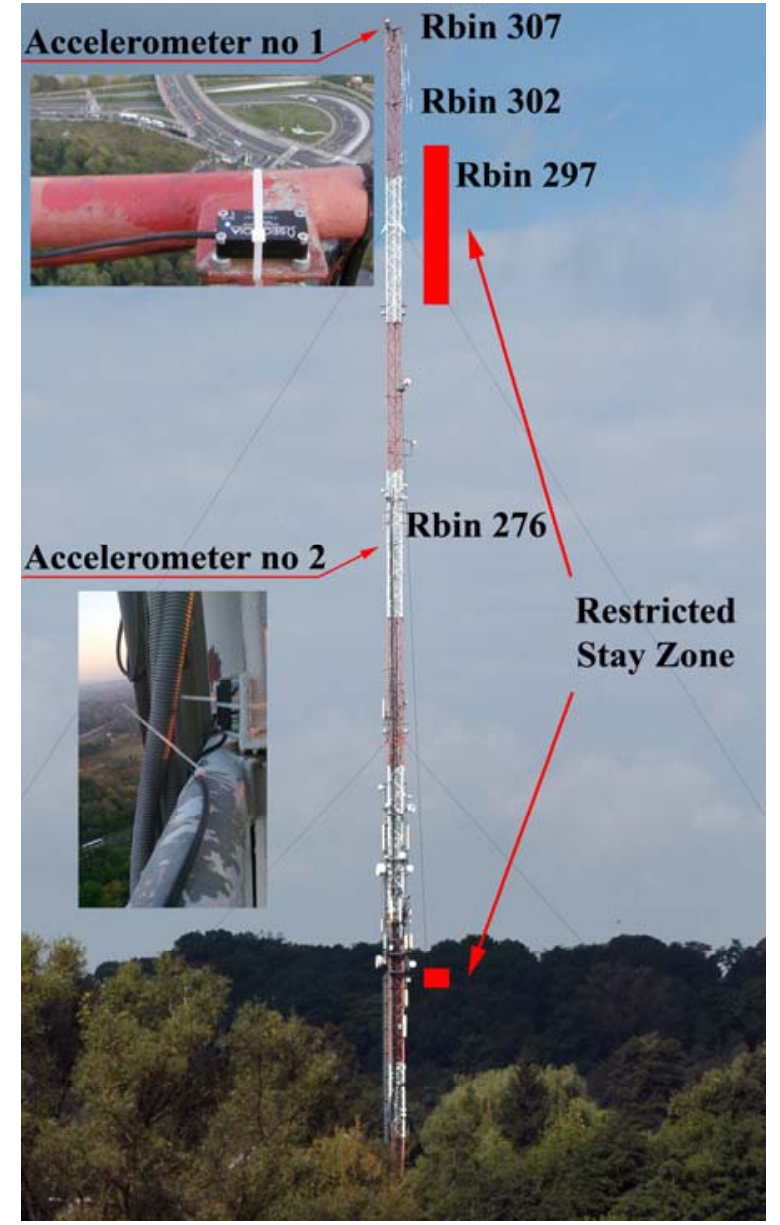

Fig. 5 Location of mounted accelerometers and the dangerous zones.

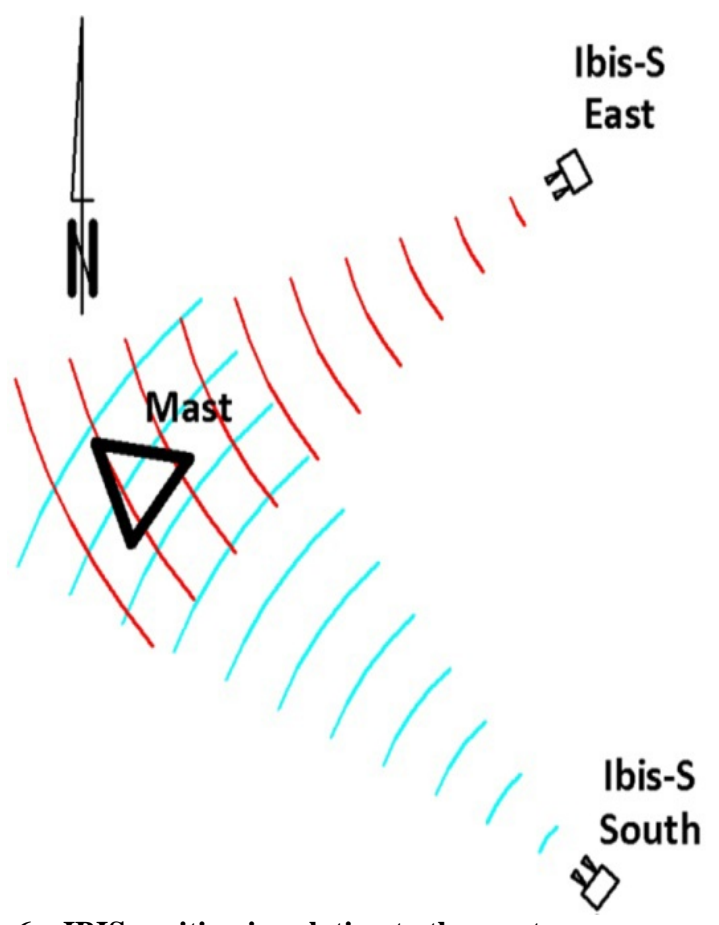

Fig. 6 IBIS position in relation to the mast.

\section{Results}

Data recording by the IBIS system was done with the frequency of $60 \mathrm{~Hz}$. Fig. 7 shows the result of data analysis made in IBIS Data Viewer software on the basis of measurement station located in southern direction from the investigated mast.

Frequencies of points vibrations are visibly consistent. In the most dangerous low frequencies range the dominant values of 0.54 (0.56), 0.90 and 0.98 $\mathrm{Hz}$ can be seen. Regardless, spectral analysis was carried out in MATLAB software. DFT (discrete Fourier transform) of data vector was computed with the FFT (fast Fourier transform) algorithm. The results are shown in Fig. 8. They are consistent with the spectrum in Fig. 7.

Spectral analysis was also carried out based on the results of acceleration measurements. For the sensor installed on the top of the mast the results are shown in Fig 9.

Although the spectral analysis for data from the IBIS system is made for displacement and for data from the accelerometer - for acceleration, the peaks of dominant frequency agree within $0.015 \mathrm{~Hz}$.

A similar comparison was performed for the point located in the middle of the central span (Rbin 276 and accelerometer No. 2). Results are presented in Figs. 10 and 11 . In this case the accordance of results is also well visible.

Recording with IBIS during observation of the chimney was carried out at a frequency of $40 \mathrm{~Hz}$. Fig. 12

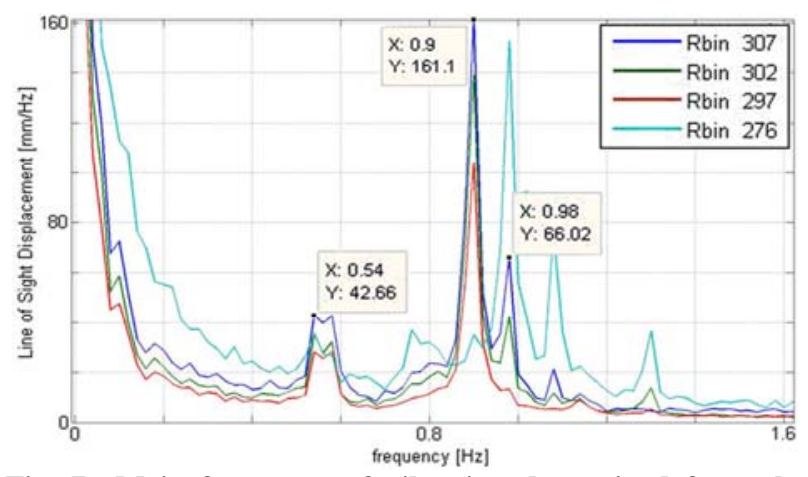

Fig. 7 Main frequency of vibration determined from the southern side. 


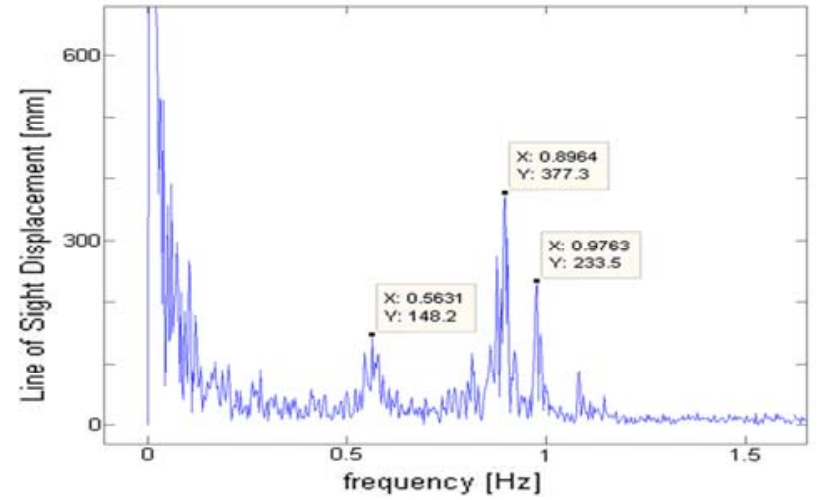

Fig. 8 DFT computed for the point Rbin 307.

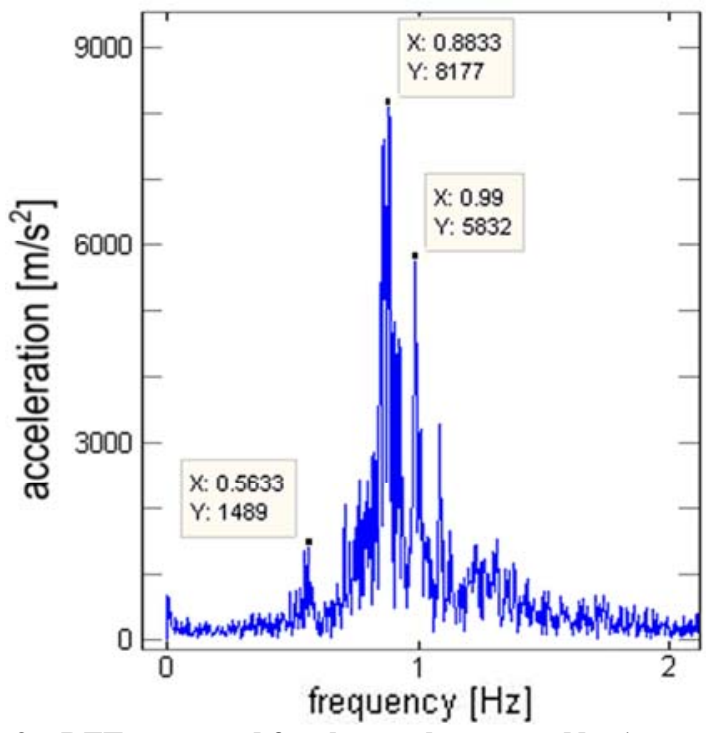

Fig. 9 DFT computed for the accelerometer No. 1.

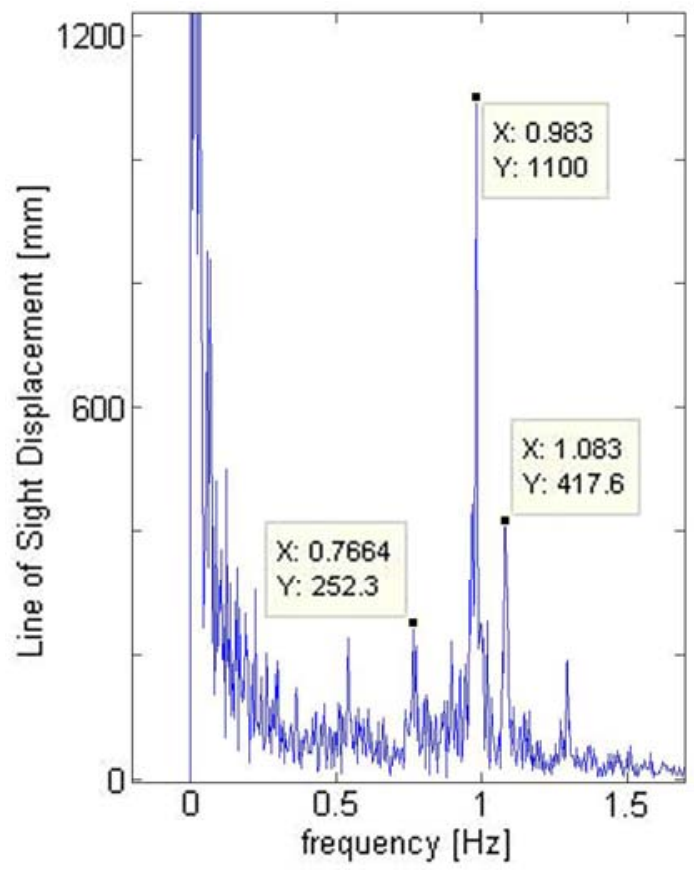

Fig. 10 DFT computed for the point Rbin 276. shows a graph of signal to noise obtained during measurement of its surface. Signal reflected from five highest steel galleries can be distinguished very clearly.

Five points selected in the radar profile, which correspond to the galleries of the chimney, were used to calculate displacement in time (Fig. 13). The highest point of the chimney was measured with a displacement of $2.5 \mathrm{~mm}$ in the direction to IBIS. The graph shows a clear oscillation of the structure core.

The frequency of vibration determined for all points has the same value of $0.16 \mathrm{~Hz}$ (Fig. 14).

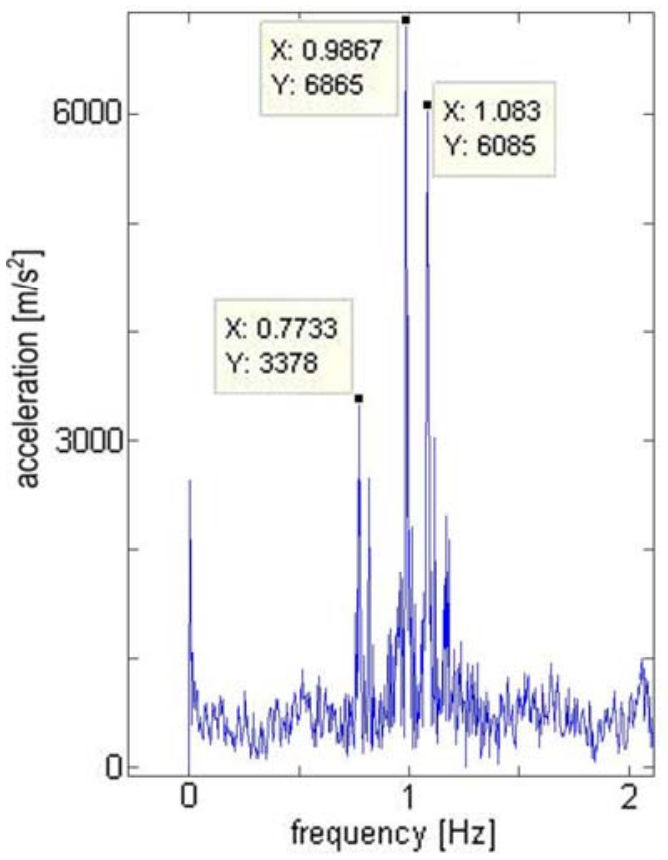

Fig. 11 DFT computed for the accelerometer No. 2.

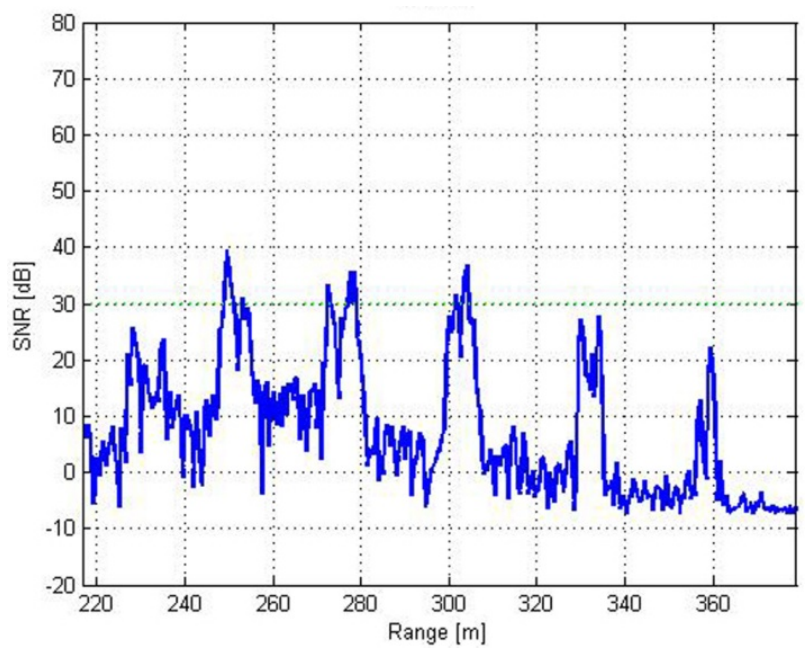

Fig. 12 The graph of signal to noise ratio obtained during observation of the chimney. 


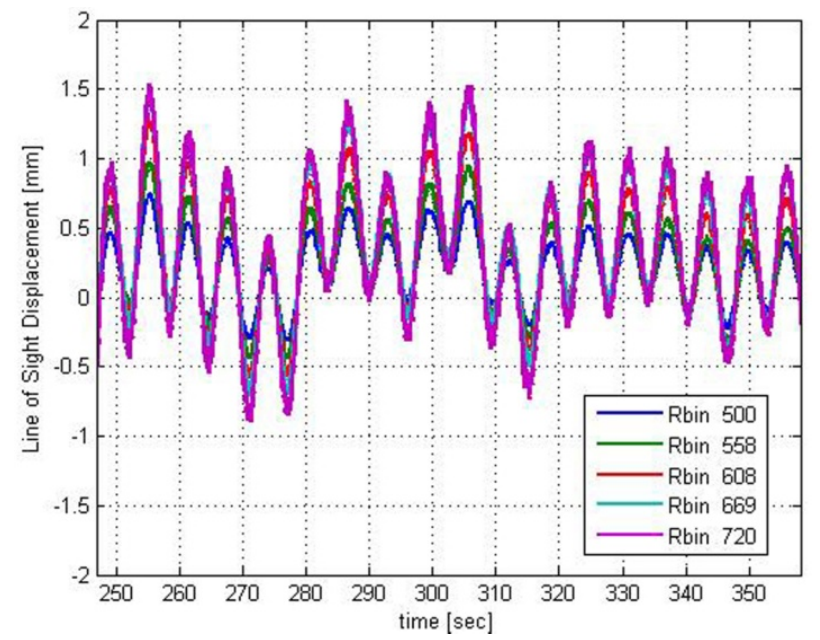

Fig. 13 Graph of displacement for the chosen points of the chimney.

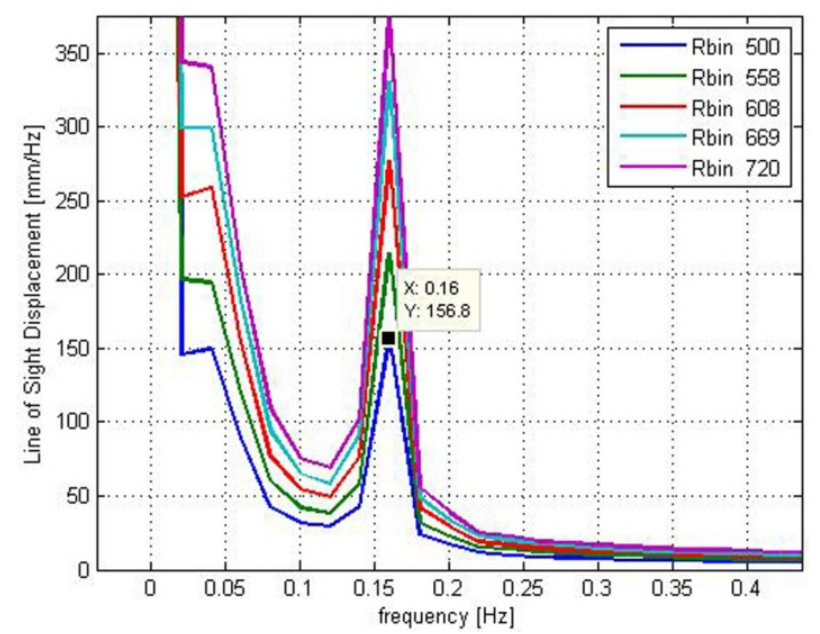

Fig. 14 Main natural frequency of chimney vibration.

\section{Conclusions}

Performed experiments allow to present the following conclusions:

- radar interferometry technique gives good results in measuring high structures. One of the main reasons is a good projection ratio of recorded displacements;

- despite the low amplitude of tested structures vibration, obtaining frequencies from the FFT based on the time series from IBIS system makes no difficulties;

- construction of tested objects allows to obtain a satisfactory radar profile, so there was no need for additional reflectors;

- despite typical intensive operating noise on the telecommunication mast, application of the wireless network for data recording from the accelerometers was possible up to 120 metres;

- results obtained from the accelerometers and the IBIS system are consistent, however, time series derived from the accelerometer are characterized by significantly higher noise.

\section{Acknowledgments}

The research was supported by the MNISW (Polish Ministry of Science and Higher Education), under grant N N526 158838.

The authors are indebted to the company TP EmiTel sp. z o.o. for providing access to the mast in Kraków.

\section{References}

[1] P. Harikrishna, A. Annadurai, S. Gomathinayagam and N. Lakshmanan, Full scale measurements of the structural response of a $50 \mathrm{~m}$ guyed mast under wind loading, Engineering Structures 25 (7) (2003) 859-867.

[2] J. M. W. Brownjohn, E. P. Carden, C. R. Goddard and G. Oudin, Real-time performance monitoring of tuned mass damper system for a $183 \mathrm{~m}$ reinforced concrete chimney, Journal of Wind Engineering and Industrial Aerodynamics 98 (3) (2010) 169-179.

[3] P. Gerstoft and A. G. Davenport, A simplified method for dynamic analysis of a guyed mast, Journal of Wind Engineering and Industrial Aerodynamics 23 (1986) 487-499.

[4] M. K. S. Madugula, Y. M. F. Wahba and G. R. Monforton, Dynamic response of guyed masts, Engineering Structures 20 (12) (1998) 1097-1101.

[5] P. D'Asdia and S. Noe, Vortex induced vibration of reinforced concrete chimneys: In situ experimentation and numerical previsions, Journal of Wind Engineering and Industrial Aerodynamics 74 (76) (1998) 765-776.

[6] C. Gentile and G. Bernardini, Output-only modal identification of a reinforced concrete bridge from radar-based measurements, NDT \& E International 41 (7) (2008) 544-553.

[7] C. Gentile, Application of radar technology to deflection measurement and dynamic testing of bridges, in: G. Kouemou (Ed.), Radar Technology, InTech., 2010, pp. 141-162.

[8] M. Pieraccini, M. Fratini, F. Parrini, G. Macaluso and C. Atzeni, High-speed CW step-frequency coherent radar for dynamic monitoring of civil engineering structures, Electronics Letters 40 (14) (2004) 907-908. 